\title{
Giant Molecular Clouds and Cluster Formation
}

\author{
Mónica Rubio \\ Departamento de Astronomía, Universidad de Chile, Casilla 36-D, \\ Santiago, Chile
}

\begin{abstract}
We will review the present knowledge of molecular cloud properties and its relation to star formation. We will discuss the evidence for cluster formation associated with giant molecular clouds, and will concentrate on recent results in our Galaxy and the Magellanic Clouds.
\end{abstract}

\section{Introduction}

Giant Molecular Clouds (GMCs) are the cool, dense phase of the interstellar medium, where star formation takes place. In our Galaxy, over 4000 GMCs have been identified with sizes of $\mathrm{R} \sim 50 \mathrm{pc}$, and masses $\mathrm{M}=10^{5}$ to $10^{6} \mathrm{M}_{\odot}$. These GMCs are known to have a great deal of complexity. They show structures in density at all scales, from $100 \mathrm{pc}$ to $10,000 \mathrm{AU}$. Stars are formed in these molecular clouds but most of its matter is sterile as stars form only in a small fraction of the mass of the cloud, typically a few \% (Leisawitz et al. 1989). Most of the mass of GMCs is in regions of low excitation and therefore photons can ionize the interstellar medium (ISM). The molecular gas is found in photodissociation or photon dominated regions (PDRs) or in regions with supersonic turbulence which allows replenishment of the ISM.

The formation of stars in the dense regions of GMCs is due to gravitational collapse. The details of how this happens depend on the physical conditions of the star-forming regions. The conditions for gravitational collapse define the mass of the star formed, the mass of the disk around it, and control the chemical properties. The theory of gravitational collapse has been developed mainly for isolated star formation. Its processes deal with induced collapse either by shock waves, collision between clouds and clumps, or a gradual process of which ambipolar diffusion and turbulence decay are the proposed mechanisms (Evans 1999). Observations of globules show that about $23 \%$ of them contain embedded IR sources of which one third show outflows that indicate a star is formed.

The formation of low-mass and high-mass stars are expected to occur in clouds with different conditions. Low mass stars can form isolated in globules and dark clouds while high mass star formation is always clustered. Near IR observations in JHK have shown the presence of multiple objects in the cores of large dark clouds. Also, deep embedded multiple sources are found towards Class 0 molecular clouds where massive $\mathrm{O}$ stars form. Usually hot cores, the previous stage of an ultra-compact (UC) H II region, show IR point source emission. 


\section{Stars are formed in clusters}

The observational evidence shows that low-mass stars form in regions of highmass star formation, where clustered formation is a rule (Elmegreen 1985). Radio continuum observations of UC H II regions (Garay and Lizano 1999; Kurtz et al. 2000) confirms that massive stars always form in clusters. Studies regarding intermediate mass stars, such as the infrared observations in JHK by Testi et al. 1999 , confirm that early Be stars are surrounded by dense clusters of lower mass "companions" while Ae stars are associated with conspicuous groups. Low-mass stars are also found to form in groups. Onishi et al. (1996) studied the Taurus cloud and found that 40 cores associated with IRAS sources had mean sizes of $0.46 \mathrm{pc}$ and masses of $23 M_{\odot}$ and the larger cores tended to have multiple objects.

Thus, most stars are born in clusters. The cluster formation and evolution influence the distribution of stars in the field and this is reflected in the initial mass function (IMF). The latest studies of the distribution of stellar masses indicate that an IMF similar to Salpeter's is derived for young embedded clusters (Meyer at al 2000; Palla and Stahler 1999). Also consistent with Salpeter's IMF are cluster forming cores, associated with pre-stellar and protostellar condensations, such as Serpens and Ophiuchi (Testi \& Sargent 1998; Motte et al. 1998). Models of cluster formation (see Elmegreen et al. 2000 and references therein; and Elmegreen, this conference) indicate that at some point the distribution of the stellar density should be different from that of the IMF. Recent observations of cores with interferometric resolution in the sub-mm range towards Serpens confirm this prediction. Testi and collaborators find that star formation is not homogeneous throughout the cores and that the "protostellar" density in the subclusters is much higher than in the mean. The authors interpret their results as the first evidence for a hierarchical fragmentation of the proto-cluster cores.

\section{GMCs and clusters in the Magellanic Clouds}

The Magellanic Clouds as the nearest external galaxies are a unique laboratory for enlarging our knowledge of GMCs and star formation.

The distribution of the molecular gas in the Large Magellanic Cloud LMC) was first determined by Cohen et al. (1988), and in the Small Magellanic Cloud (SMC) by Rubio et al (1991). About 40 and 7 molecular complexes, respectively, were identified at a resolution of $140 \mathrm{pc}$. These complexes with sizes of $\mathrm{R} \sim 200$ pc and masses $\sim 10^{6} M_{\odot}$ were found close to $\mathrm{H}_{\text {II }}$ regions and in areas where the $\mathrm{N}(\mathrm{H} \mathrm{I})$ column density exceeded $10^{21} \mathrm{~cm}^{-2}$ and $10^{22} \mathrm{~cm}^{-2}$ in the LMC and SMC, respectively. No correlation with SNRs was found. The total molecular gas mass derived from these surveys was a relative small fraction of the total gas mass (less than 30\%). The physical properties of the molecular clouds have been obtained from high angular resolution of the molecular complexes with the SEST telescope (see Rubio 1999.) At a linear resolution of $10 \mathrm{pc}$ molecular complexes were resolved into clouds of typical sizes $\mathrm{R}=20 \sim 30 \mathrm{pc}$ with masses $\mathrm{M} \sim 10^{4}-10^{5} \mathrm{M}_{\odot}$.

In the last 3 years, the NANTEN telescope has performed a new and more sensitive $\mathrm{CO}(1-0)$ survey of the Magellanic Clouds. This survey covers $6^{\circ} \times 6^{\circ}$ 


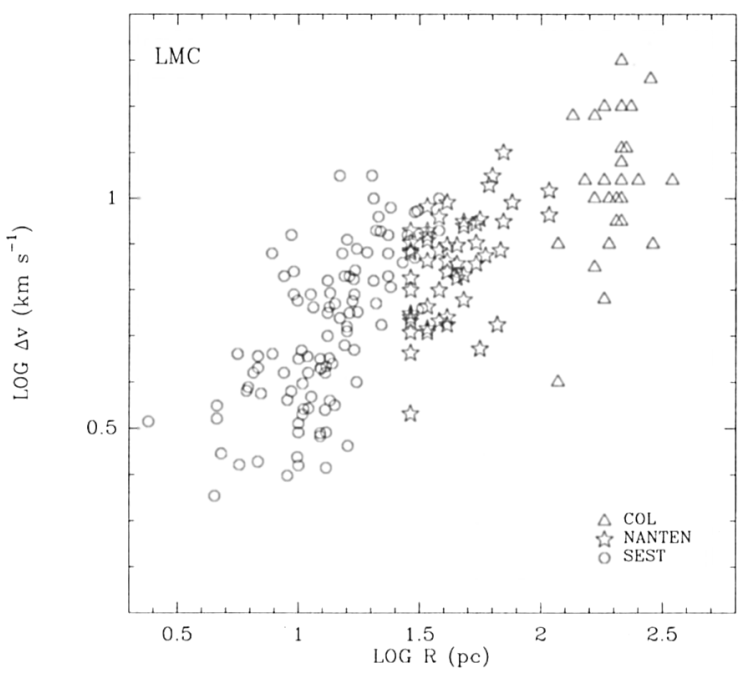

Figure 1. Radius versus line width plot for LMC molecular clouds as determined from different linear scale resolution. A least square fit to all points gives $\Delta v \sim R^{0.4}$

in the LMC and about 1 square degree in the SMC at a 40 pc linear resolution. More than 100 and 20 molecular clouds in the LMC and SMC, respectively, have been fully mapped. In the LMC, the molecular clouds show masses $\mathrm{M} z$ $5 \times 10^{4} M_{\odot}$. Cloud properties were derived for 55 clouds and these showed ranges of sizes $R \sim 30-100 \mathrm{pc}$, masses $\mathrm{M} \sim 10^{4}-10^{6} M_{\odot}$, and line widths $\Delta v=4-13$ $\mathrm{km} \mathrm{s}^{-1}$ (Fukui et al. 1999). The mass distribution for the LMC molecular clouds is found to be $\mathrm{dN} / \mathrm{dM} \sim-2$ and could be indicative of the fractal structure of molecular clouds in this galaxy.

Although the GMCs in the Magellanic Clouds are less luminous in $\mathrm{CO}$ than the GMCs in our Galaxy, they do show certain similarities. They follow a similar $\Delta v \sim R^{\gamma}$ relationship as that found for Galactic molecular clouds. Figure 1 shows a plot of the sizes versus line width for molecular clouds in the LMC as determined from observations done with different resolution. The SEST observations (circles), the NANTEN observations (stars), and the Columbia observations (triangles) have linear resolutions of 10,40 and $140 \mathrm{pc}$, respectively. A least square fit to all the data points give $\Delta v \sim R^{\gamma}$, with $\gamma \sim 0.4$. For GMCs in our Galaxy, $\Delta v \sim R^{0.5}$, (Solomon et al. 87). Also, the molecular clouds in the LMC are globally well correlated with $\mathrm{H}$ II regions and young clusters. A detailed comparison of GMCs to stellar clusters and OB associations has been made over the whole LMC (Yamaguchi et al. 2001; Mizuno et al., these proceedings). They find that the GMCs are actively forming stars and clusters. A large fraction of the GMCs are found to be associated with young clusters and an even larger fraction is found associated with $\mathrm{H}$ II regions. All clusters 
younger than $30 \mathrm{Myr}$ are found to be associated with $\mathrm{H}$ II regions. Only 15 of the 55 GMCs used in this study were found not to be associated with any young object. These GMCs show the same properties as those associated with H II regions. In addition, compact groups of the youngest clusters are found at or near the peak of the GMCs, e.g, N159, N44, while looser groups of clusters tend to lie away or at the edge of the GMCs. They suggest that star clusters form in compact groups and scatter as they evolve. They propose an evolutionary time-scale for the GMCs. in which in a time span of 10 Myrs, GMCs form stars in the first few My, they form clusters in the next few My and they dissipate in the next few My. The formation of massive compact clusters could be due to the interaction of massive GMCs which produces the conditions to start the star forming process in a GMC. This could be the case for 30 Doradus which lies in the intersection of two superbubbles.

\section{Star Formation in the Magellanic Clouds}

We have been investigating the process of star formation in the GMCs in the Magellanic Clouds in the near IR. We have done a deep JHKs survey of a large selection of molecular clouds and star forming regions in both the LMC and SMC. Our results show that young stellar objects (YSOs) are found embedded in the molecular regions associated with star formation in the Magellanic Clouds. Moreover, evidence for a second generation of stars, probably triggered or induced by the first event is found towards 30 Doradus (Rubio et al. 1998) and N11 (Barbá \& Rubio 2001) in the LMC and N66 in the SMC (Rubio et al. 2000). High sensitivity $\mathrm{CO}(2-1)$ observations towards these regions show that dense clouds of molecular gas survive the action of the strong UV radiation field of the massive early $\mathrm{O}$ type stars. We found morphological structures similar to those found in star-forming regions in the Galaxy, such as elephant trunks and pillars in the PDR regions.

We recently finished our studies of N11B in the LMC (see Barbá and Rubio, these proceedings) and the morphological similarities with some high-mass Galactic star-forming regions are striking. A cluster of embedded IR sources is found towards the peak of the molecular cloud. In this region an $\mathrm{OH}$ maser has been reported. Narrow band images obtained with HST/WFPC2 show that there is a pillar shining in OIII with the IR embedded sources in its interior. The PDR is excited by two $\mathrm{O}$ stars located in the outer region of the molecular cloud. N11 is the second largest $\mathrm{H}$ II region in the LMC after 30 Doradus and it is suggested that is in a later stage of evolution than 30 Doradus.

In a previous study, we found in the brightest H II region in the SMC, N66, that induced star formation at the border of the molecular cloud was taking place (Rubio et al. 2000). A PDR region, well delineated in the $\mathrm{H}_{2} \mu \mathrm{m}$ emission line, was observed at the interface between the molecular cloud and the UV radiation field. This strong field is produced by the central cluster NGC 346 which contains more than $35 \mathrm{O}$ stars. Strong knots of $\mathrm{H}_{2}$ emission appeared towards the $\mathrm{CO}(2-1)$ densest regions with embedded IR sources .

More spectacular is the case of the giant $\mathrm{H}$ in region 30 Doradus, energized by a dense compact cluster of more than $65 \mathrm{O}$ stars of which more than 30 are of O3 type. This region is not only forming a second generation of stars seen as 
YSO-like embedded sources (Rubio et al. 1998), but it is likely forming several clusters. The ground based YSOs have been resolved into multiple components by HST/NICMOS images (Walborn et al. 1999, Brandl et al. 2001). The UV radiation field is interacting with the molecular gas creating PDR regions in the borders of the dense gas. These regions are well seen in the narrow line images obtained with HST. Also, $\mathrm{H}_{2}$ molecular knots and clumps are seen at $2.12 \mu$ which are coincident with dense CO structures (Rubio 1999). The morphology again reveals the presence of pillars, trunks, dark globules and volcanos which are all illuminated in the direction facing the central core, R136. In all these cases, sensitive $\mathrm{CO}(2-1)$ observations have revealed the presence of compact dense regions of molecular gas, remnants of the parental molecular cloud.

\section{Summary}

In our galaxy, we have observational evidence that most stars form in groups or clusters at the interior of the dense regions in the GMCs. It is still a matter of study how massive stars and low mass stars form in the same region, while a clearer picture has emerged for regions of low-mass star formation. In the Magellanic Clouds we have found that GMCs are an important component of the interstellar medium and that these GMCs have molecular masses as large as $10^{6} M_{\odot}$ although they are less luminous in CO than their Galactic counterparts. Recent studies have shown that young clusters and $\mathrm{H}$ II regions are closely associated with GMCs and that an evolutionary process from GMCs to young cluster formation takes about $10 \mathrm{My}$. In addition, our studies of the stellar content of star forming regions in the LMC and SMC shows that these regions have several episodes of massive star formation in the lifetime of a GMC, as dense regions of molecular gas survive the action of photodissociation and photoevaporation of a strong radiation field. In particular the 30 Doradus region, due to its large amount of mass, its high number of stars and dense compact core, could be the case of formation of a compact cluster. Massive young clusters in external galaxies show a compact core surrounded by a massive halo. 30 Doradus is possibly such a massive cluster and we are witnessing that the formation of the central massive core takes place first and the massive halo later. The central core is 2 My older than the outer halo (Maiz-Apellaniz 2002, these proceedings).

With the advent of $8 \mathrm{~m}$ class telescopes and sensitive detectors in the NIR we will be able to study the embedded population in the Magellanic Cloud molecular clouds to masses down to $1 M_{\odot}$ from the ground and determine the IMF to these masses in these clusters. In the future, NGST and ALMA will allow us to learn about star clusters and GMCs in even more distant galaxies.

Acknowledgments. M.R acknowledges the SOC for inviting her to speak at this Symposium. This work has been funded by FONDECYT grant $N^{\circ} 1990881$ and FONDECYT INTERNATIONAL grant $N^{o} 7900042$.

\section{References}

Barbá, R. \& Rubio, M. 2002, in preparation

Brandl, W. et al. 2002, AJ, in press 
Cohen et al., 1988, 331, L95

Evans, N. 1999, ARA\&A, 37, 311

Elmegreen B. 1985, in Protostar and Planets II, eds. D.C. Black, M.S. Matthews, (Tucson: Univ. Arizona Press), 35

Elmegreen, B. et al. 2000, in Protostar and Planets IV, University Arizona Press, eds. V.G. Manning, A.P. Boss, and S.S. Russell, 179.

Fukui et al. 1999, PASJ, 51, 745

Garay, G. \& Lizano, S. 1999, PASP, 111, 1049

Kurtz et al 2000, in Protostar and Planets IV, University Arizona Press eds.

V.G. Manning, A.P .Boss, and S.S. Russell, in press

Leisawitz, D., Bash, P.N., Thaddeus, P. 1989, ApJS 70, 737

Maiz-Apellaniz, J. 2002, these proceedings

Meyer et al 2000, in Protostar and Planets IV, University Arizona Press eds.

V.G. Manning, A.P. Boss, and S.S. Russell, in press

Mizuno, N. et al. 2002, these proceedings

Motte et al. 1998, A\&A 336, 150

Palla, F. \& Stahler, S. 1999, ApJ 525, 772

Rubio 1999 in New Views of the Magellanic Clouds, eds. Y.H.Chu, N. Suntzeff, J.Hesser, \& D. Bohlender, (San Francisco:ASP), 67

Rubio et al. 1991, ApJ, 368, 173

Rubio et al. 1998, AJ, 116, 1705

Rubio et al. 2000, A\&A, 359, 1139

Solomon et al. 1987. ApJ, 319, 730

Testi \& Sargent 1998, ApJ, 505, L39

Walborn et al. 1999, AJ, 117, 225

Yamaguchi et al. 2001, PASJ, 53, 985

\section{Discussion}

H. Zinnecker: I am very interested in your $2^{\prime} \times 2^{\prime}$ infrared JHK images of N159 and N11. As you know, these regions were also studied with WFPC2/HST by Heydari-Malayeri et al. in the optical, in particular in the I band. Which new feature did you see in JHK data which were not seen in the I band already?

M. Rubio : The data I have is very deep: to $K_{s} \sim 18$ and therefore it shows several new embedded IR sources which are not visible in the I band HST images since they are towards dense regions of molecular gas.

S. Silich: Could you please comment on the shapes of giant Molecular Clouds? Are GMCs on average round, or do you have indications that they are elongated?

M. Rubio : In general, the shapes of the CO clouds as seen from the NANTEN survey are mainly round but this could be due to the beam size of about 2 arcmin. I have not looked into this detail in the SEST data. 